\title{
W-FYD: A Wearable Fabric-Based Display for Haptic Multi-Cue Delivery and Tactile Augmented Reality
}

\author{
Simone Fani ${ }^{\circledR}$, Student Member, IEEE, Simone Ciotti ${ }^{\odot}$, Student Member, IEEE, \\ Edoardo Battaglia, Student Member, IEEE, Alessandro Moscatelli ${ }^{\circledR}$, and Matteo Bianchi ${ }^{\odot}$, Member, IEEE
}

\begin{abstract}
Despite the importance of softness, there is no evidence of wearable haptic systems able to deliver controllable softness cues. Here, we present the Wearable Fabric Yielding Display (W-FYD), a fabric-based display for multi-cue delivery that can be worn on a user's finger and enables, for the first time, both active and passive softness exploration. It can also induce a sliding effect under the finger-pad. A given stiffness profile can be obtained by modulating the stretching state of the fabric through two motors. Furthermore, a lifting mechanism allows to put the fabric in contact with the user's finger-pad, to enable passive softness rendering. In this paper, we describe the architecture of W-FYD, and a thorough characterization of its stiffness workspace, frequency response, and softness rendering capabilities. We also computed device Just Noticeable Difference in both active and passive exploratory conditions, for linear and non-linear stiffness rendering as well as for sliding direction perception. The effect of device weight was also considered. Furthermore, performance of participants and their subjective quantitative evaluation in detecting sliding direction and softness discrimination tasks are reported. Finally, applications of W-FYD in tactile augmented reality for open palpation are discussed, opening interesting perspectives in many fields of human-machine interaction.
\end{abstract}

Index Terms-Wearable haptics, softness rendering, sliding stimuli, haptic multi-cue delivery, tactile augmented reality, psychophysics

\section{INTRODUCTION}

W EARABLE haptic systems (WHSs) represent a new category of devices that can be easily worn by users. Such devices have gained an increasing attention in recent years, since they enable a more natural and effective human robot interaction (HRI). Indeed, WHSs can be carried around and integrated into everyday life, with minimal or no constraints to human motion [1] and ideal applications related (but not limited) to assistive technologies [2], virtual reality [3], affective touch [4] and tele-manipulation with remote robotic systems [5]. Among the different strategies used to design wearable/portable haptic devices, we can

- S. Fani and S. Ciotti are with the Research Center "Enrico Piaggio", University of Pisa, Largo Lucio Lazzarino 1, Pisa 56126, Italy, and the Department of Advanced Robotics, Istituto Italiano di Tecnologia, via Morego, 30, Genova 16163, Italy. E-mail: \{simonefani89, ciottisimone88\}@gmail.com.

- E. Battaglia is with the Research Center "Enrico Piaggio", University of Pisa, Largo Lucio Lazzarino 1, Pisa 56126, Italy.

E-mail:e.battaglia@centropiaggio.unipi.it.

- A. Moscatelli is with the Department of Systems Medicine and the Center of Space Bio-Medicine, University of Rome "Tor Vergata", Rome 00173, Italy, and the Laboratory of Neuromotor Physiology, IRCCS Santa Lucia Foundation, Rome 00179, Italy. E-mail: a.moscatelli@hsantalucia.it.

- M. Bianchi is with the Research Center "Enrico Piaggio", University of Pisa, Largo Lucio Lazzarino 1, Pisa 56126, Italy, and the Department of Information Engineering, University of Pisa, via G. Caruso, 16, Pisa 56122, Italy. E-mail: matteo.bianchi@centropiaggio.unipi.it.

Manuscript received 14 Feb. 2017; revised 16 May 2017; accepted 18 May 2017. Date of publication 9 Aug. 2017; date of current version 15 June 2018.

(Corresponding author: Matteo Bianchi.)

Recommended for acceptance by H. Z. Tan.

For information on obtaining reprints of this article, please send e-mail to: reprints@ieee.org, and reference the Digital Object Identifier below.

Digital Object Identifier no. 10.1109/TOH.2017.2708717 mention vibration-based systems [2], pin-arrays [6], force applications at one or more contact points [7]. These devices can be worn on different body parts such as arm [1], [8], wrist [9], foot [10] and finger [3], [11] - for a review on these topics, the reader is invited to refer to [11].

Considering human finger as the privileged channel for discriminative touch, various wearable technological solutions for tactile stimuli delivery can be found in literature.

In [3], authors described a 3 Degrees Of Freedom (DOF) wearable system for modulating skin stretch. A tactor-based display to render planar displacement was presented in [12]. In [13], [14], authors proposed a WHS that consists of two motors to convey both normal and tangential forces, thus simulating weight sensation. A similar device—namely hring-endowed with two servo motors, which move a belt placed in contact with the user's finger, conveys both normal and shear force to the skin [15]. A parallel mechanism with three actuated DOFs, which is able to render contact forces with general direction was described in [11]. Haptic Thimble in [16] combines wearable rendering of surface orientation with fast transient and wide frequency bandwidth tactile cues for the exploration of virtual surfaces in virtual environment.

All these solutions are very promising and increase user's performance in human-machine interaction: however, none of these devices takes into account softness rendering. This is surprising, considering that softness is basically a touchrelated property, representing one of the most accessible sources of information after the initial phases of contact [17], [18]. This fact has motivated the attention in the investigation 


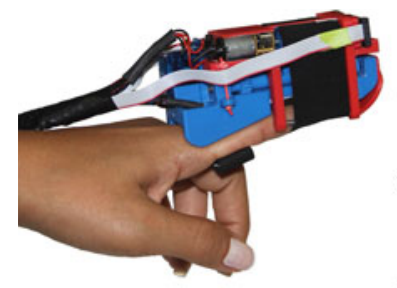

(a)

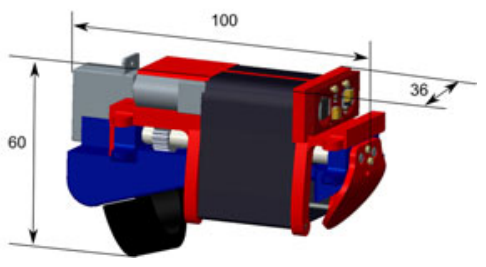

(b)
Fig. 1. W-FYD Prototype. In figure are shown (a) the W-FYD worn by an user and (b) the CAD design with dimensions in $\mathrm{mm}$. The total mass of the device is $100 \mathrm{~g}$.

of haptic perception of compliance (e.g., [19], [20]) and the effort in developing effective haptic softness displays in applications such as tele-operation [21].

With this as motivation, in our previous conference paper [22], we presented a wearable device, hereinafter referred to as W-FYD (Wearable-Fabric Yielding Display, see Fig. 1a). W-FYD enables, for the first time, to convey controllable softness information to the user's finger-pad, allowing both active and passive haptic exploration [20]. Furthermore, stimuli related to tangential sliding can be also delivered. W-FYD represents the wearable version of a grounded softness display we described in [23], [24], [25], [26], which can reproduce different stiffness levels through the control of the stretching state of an elastic fabric. It is worth to note that the device in [13] has a structure similar to the one of W-FYD. In [13], two DC motors can stretch a belt around user's fingerpad to convey force and gravity cues. Furthermore, if independently controlled, they can also deliver tangential force information. For the design of our device, we have adopted a similar structure but we have implemented an additional Degree Of Actuation (DOA) trough a servomotor and lifting mechanism. This additional DOA puts the fabric in contact with the user's finger-pad, which is still, while fabric stiffness is varied through the two DC motors. In this manner, we can convey different stiffness cues in an independent fashion from the force experienced by the user, thus enabling, for the first time, passive softness exploration. In [27], a similar solution was described, but it is not suitable for wearable implementation.

There is also an additional asset in W-FYD design. Leveraging upon device wearability and fabric deformability [28], we can use W-FYD to superimpose tactile cues without compromising the sensation derived from direct touch with external items. These characteristics can be very useful to enrich haptic experience in augmented reality (AR), laying the foundations of a new paradigm for humanmachine interaction, i.e., tactile augmented reality. We will report an implementation of such a paradigm in Section 6, where we describe the usage of a modified version of W-FYD to reproduce artery pulse in an AR simulator for laparoscopic cholecystectomy [29].

In this work, we further develop the approach and investigations in [22] by: (i) performing a more accurate and thorough characterization of the stiffness workspace of W-FYD, and device capabilities in rendering linear and non linear softness; (ii) reporting the identification of W-FYD system, in terms of transfer function between control inputs to the servo-motor and measured output displacements of the

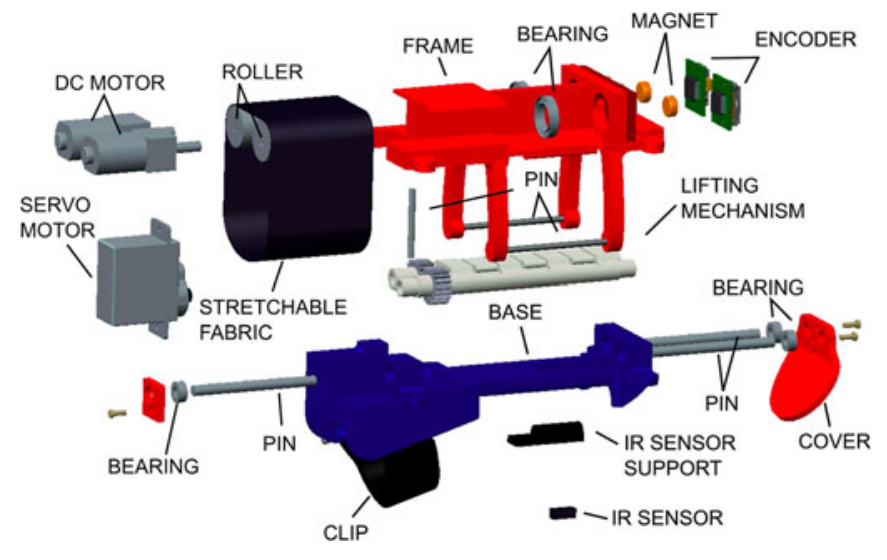

Fig. 2. W-FYD exploded view.

lifting mechanism; (iii) carrying out a completely new and exhaustive device validation through human experiments. More specifically, we have computed W-FYD Just Noticeable Difference (JND) for linear and non-linear softness discrimination, in both active and passive mode, with and without a weight support. The latter was used to understand the effect of device weight in the perception elicited in users. Furthermore, we have also computed JND for sliding stimuli, still taking into account the effect of the weight. Finally, we run an experiment where the device was controlled to simulate three levels of nonlinear stiffness and to convey sliding stimuli to participants, who were asked to perform a relative recognition task and complete a subjective quantitative evaluation through a Likert-type scale, which focused on wearability and the effectiveness of W-FYD in stimuli delivery.

Applications of W-FYD in tactile augmented reality environment [29] for surgical training are also reported, together with conclusions and future works.

\section{System ARChitecture}

The design of W-FYD (Fig. 1b) is a wearable evolution of the grounded FYD-2 [26]. As in the FYD-2, a layer of isotropic elastic fabric (Superbiflex HN by Mectex S.P.A) is used in the device as interaction surface for the user.

The device is placed over the user's finger, and fixed to it with an elastic clip that prevents rotation and ensures stability. The clip was designed to prevent rotation around the finger while the finger is held horizontally (also in our Augmented Reality applications). In future works, we will investigate the fixing mechanism also for other orientations of the device, together with a more exhaustive characterization of users fatigue, and workspace. Fig. 2 shows an exploded view of the W-FYD, which consists of two main parts: the base, which is fixed and contains a lifting mechanism; and the frame, hosting two DC motors that independently move two rollers attached to the elastic fabric, thus varying its stiffness. Two pins are connected to the frame through supports, ensuring that a planar surface is offered for the fingertip to interact with when the fabric is pulled.

Two different modes of interaction can be obtained from this device: a passive mode, where the user receives a mechanical stimulation from the lifting mechanism pressing the fabric against the finger-pad, and an active mode, where 


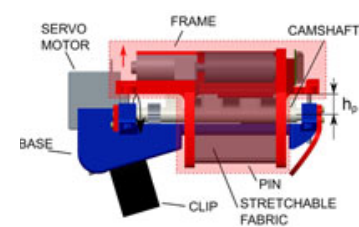

(a)

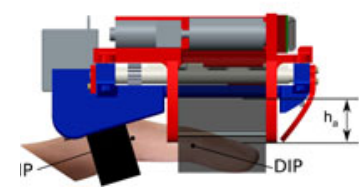

(d)

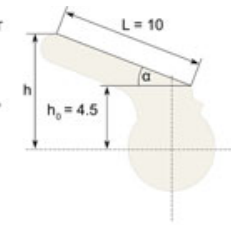

(b)

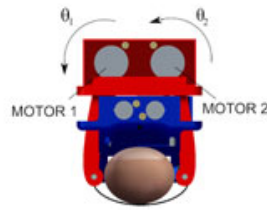

(c)

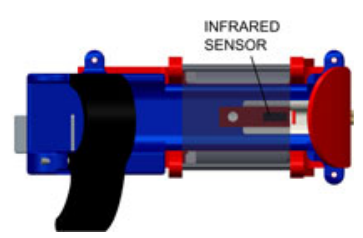

(e)

Fig. 3. W-FYD: Mechanical description. (a) Lifting mechanism and finger interaction in passive mode (the moving components are hilighted in red). (b) Lifting mechanism: Cam profile. Dimensions in $\mathrm{mm}$. (c) Definition of the angles relative to the two motors. (d) Finger interaction in active mode. (e) Infrared sensor placement.

the finger actively probes the interaction surface for softness. Note that in the passive mode the finger is still and the lifting mechanism puts the fabric in contact with the fingerpad, thus conveying only tactile cues to the skin [20]. In the active mode, the device is attached to the back of the finger, hence the only movement the user can perform is the flexion of the distal phalanx to indent the fabric, as shown in Fig. 3d.

The distinguishing feature of the W-FYD is that, since the fabric stretching is actuated separately from the lifting mechanism, the stiffness of the fabric can be changed independently and is decoupled from the force exerted on the user in the passive mode. This is an important difference respect to previously proposed designs, e.g., [13], [30], where the force increases as the sheet is wrapped around the finger, thus not allowing to independently control force and stiffness.

The lifting mechanism on the base is actuated by a servomotor (HS-5055MG Servo by Hitec), and consists of two camshafts connected by gears. The frame part rests over the camshaft, and pins are used to constrain its motion respect to the base to a vertical movement. As the lifting mechanism is moved by the servo motor, the frame moves upward, together with all the parts attached to it (Fig. 3a). This causes the surface of the fabric, whose stretching state is independently regulated through the control of the DC motors, to be pressed against the finger-pad. In particular, with reference to Figs. $3 a$ and $3 b$, the relationship between the angle of the servomotor $\alpha$ and height variation of the frame $h_{p}$ is given by the equation

$$
h_{p}=h_{0}+L \sin \alpha,
$$

where $L$ is the length of the arm of the shaft, $\alpha$ is the angle between the arm of the shaft and the horizontal plane, and $h_{0}$ is the radius of the shaft. This equation makes it possible to determine $h_{p}$ from the movement of the servomotor. We set as maximum value for $h_{p} 10 \mathrm{~mm}$, which was heuristically chosen to enable a compelling softness rendering in passive mode while preserving user's comfort. This value was set in preliminary experiments.

The two DC motors (Pololu 298:1 Micro Metal Gearmotor) are attached to the frame and, independently, positioncontrolled with readings of two absolute magnetic encoders (16 bit magnetic encoder AS5045 by Austria Microsystems). Changes in the stretching state of the fabric are obtained by suitably controlling the relative angle between the two DC motors, causing different levels of stiffness to be perceived by the user. More details on device stiffness workspace are reported in the following Section.

In the active control mode, the user can explore different stiffness levels, by moving the inter-phalengeal (IP) and distal-interphalangeal (DIP) joints (Fig. 3d). This is made possible thanks to the clip mechanism, which ensures the stability of the worn system, while preserving user's capability to actively interact with the fabric. In this case, the indentation $h_{a}$ produced by the finger is measured by one contact-less infrared (IR) sensor (Avago HSDL-9100 analog distance sensor with detection range from 0 to $60 \mathrm{~mm}$ ). The IR sensor is attached to the base (Fig. 3e).

Finally, because of the presence of the two independently controlled DC motors, W-FYD is endowed with an additional translational degree of freedom, which can induce a sensation of sliding/slipping on the user's fingertip. In this case, the user wears the system and the DC motors are synchronously moved, so that the fabric slides against the user's finger (which is still), right and left.

A custom made electronic board (PSoC-based electronic board with RS485 communication protocol https:// www.naturalmachinemotioninitiative.com/) controls motor positions-based on the readings of the encoders-and the servo motor, and enables to acquire the measurements from the IR sensor. The entire cycle works at a frequency of $200 \mathrm{~Hz}$. More details on the system control methods are provided in the following Sections. All the structural parts of W-FYD are in ABSplus-Stratasys, rapid prototyping material.

\section{System Characterization and Control}

This Section discusses the characterization of the system in terms of the reproducible stiffness workspace. We also describe the control modes we have implemented for both active and passive stiffness delivery, including experimental validation of W-FYD capabilities in non linear softness rendering. Finally, we report on the identification of W-FYD system, in terms of transfer function between control inputs to the servo-motor and measured output displacements of the lifting mechanism.

\subsection{Characterization}

In order to correctly use the W-FYD, the first mandatory step is the characterization of its stiffness workspace, in terms of force $(F$, in $\mathrm{N})$ and indentation $(\delta$, in $\mathrm{mm})$, similar to what we did in [22], [26].

In this work, we used the Zwick/Roell Z005 Table Top materials testing machine (by Zwick/Roell, Germany). ${ }^{1}$ This machine uses an indenter (see later in the text) to induce a controllable vertical displacement on the fabric, while normal force information is recorded. We performed

1. For further information on the testing machine, please see https://www.zwick.com/ 


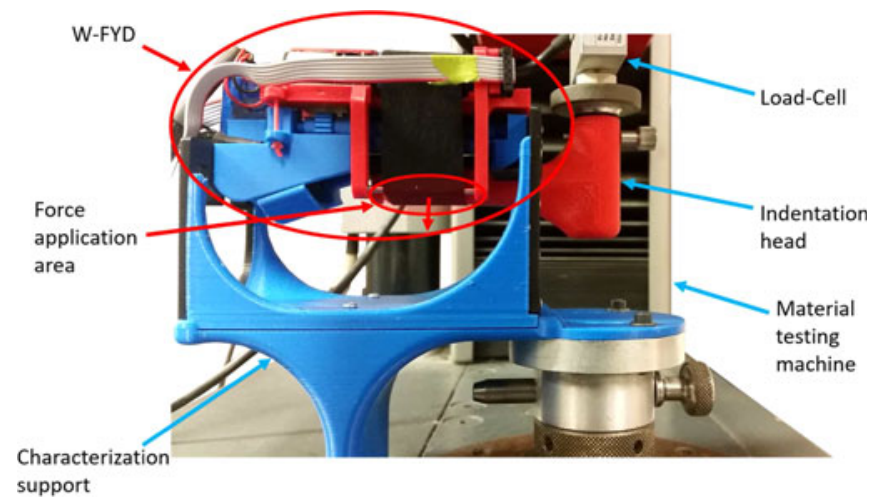

Fig. 4. Characterization setup.

this characterization for different stretching states of the fabric corresponding to different angular positions of the two DC motors. These positions are hereinafter referred to as $\theta_{1}$ and $\theta_{2}$, respectively. With reference to Fig. 3c, we assigned $\theta_{2}=-\theta_{1}=\theta$, and considered $\theta$ ranging from 0 to 100 degrees, with 10 degrees of incremental step.

The characterization system can control the indentation through a linear actuator with an accuracy of $1 \mu \mathrm{m}$, while normal contact force is measured with an accuracy of $1 \mathrm{mmN}$. Motivation for this new characterization is twofold. First, the need of relying on a very accurate and reliable testing system. Second, in the new version of the device, we have changed the dimensions of the fabric actuated and wrapped around the DC motors to provide the interaction surface, from $150 \times 20 \mathrm{~mm}$ to $300 \times 25 \mathrm{~mm}$. This resulted in a different zero position for the motors w.r.t. [28] as well as in different softness characteristics. This choice is mainly motivated by a trade-off between stiffness workspace and the need of having longer duration for the sliding stimuli to increase user's immersiveness in applications for augmented reality - which is one of the ideal application fields of W-FYD, as discussed later.

To perform the characterization, the W-FYD was mounted on a suitable characterization support printed in ABS and fixed under the indentation machine. An indenter (or indentation head) was also printed in ABS and applied to the machine linear actuator in order to provide controllable indentation steps to the fabric, while the interaction force was recorded by the testing machine through a load cell. The overall characterization setup is reported in Fig. 4. The indentation head consists of an half-cylinder (with radius $\mathrm{r}$ equal to $8.5 \mathrm{~mm}$ and length $\mathrm{L}$ equal to $40 \mathrm{~mm}$ ) and a semi-sphere of same radius fabricated in rapid prototyping material, to model the last phalanx of a human finger as in [26]. As also discussed in [26], ideally the differences between the indentation head (which is a non-compliant object) and human fingertip (which is a compliant object) should be considered during the characterization procedure; however, since the deformation of the fingertip that interacts with the fabric is small, the approximation of the indenter with a non-deformable object is still acceptable. Indeed, given the range of stiffness that can be reproduced by the device, the deformation of the fabric is usually larger than the one produced on the finger-pad-further details are provided later in this Section. This observation is also motivated by a recent characterization of finger-pad mechanical response performed by our group [31].

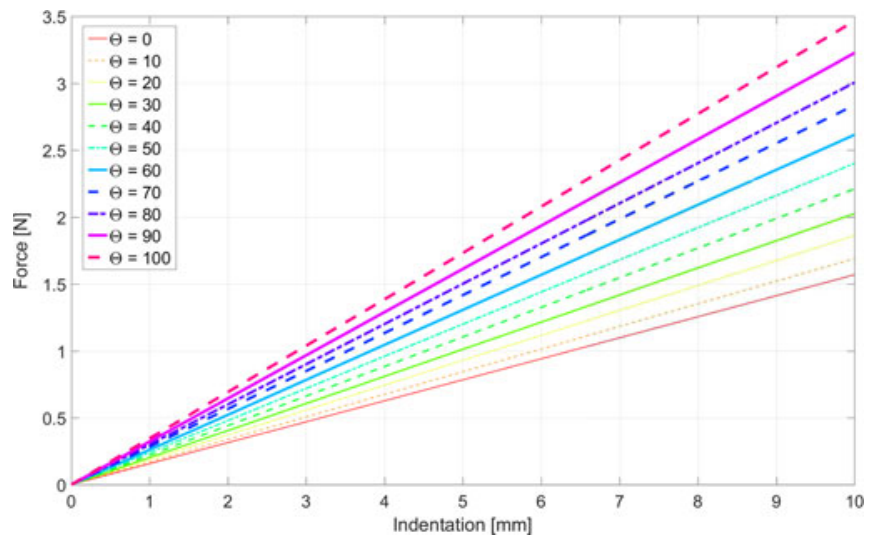

Fig. 5. Force indentation characterization.

The indentation head was moved using a single indentation step of $10 \mathrm{~mm}$, with an indenting velocity of $0.5 \mathrm{~mm} / \mathrm{s}$. We activated an emergency stop condition via software for the testing machine, which was automatically activated when the value of the indentation forces was higher than $10 \mathrm{~N}$. This choice was made to avoid damages to the W-FYD device.

As we did in [26], [28], we chose a linear interpolation for the force/indentation characteristics over the different motor positions (goodness of fit $R^{2}>0.95$ ), and reported in Fig. 5. Thus, we can write the force/indentation characteristics as function of $\theta$ as $F=\sigma(\theta) \delta$, where $F$ is the indenting force while $\delta$ is the indentation produced on the fabric. $\sigma(\theta)$, i.e., the function that provides the stiffness coefficient for a given value of $\theta$ can be expressed as

$$
\sigma(\theta)=a e^{b \theta}+c e^{d \theta}=1.543 e^{0.002909 \theta}-1.388 e^{0.002126 \theta},
$$

with a goodness of fit of $R^{2}=0.9994$.

\subsection{Softness Rendering}

W-FYD can reproduce linear stiffness relying on the characterization reported in the previous section. In this case, motor position $\theta$ associated to a given linear stiffness coefficient can be obtained by inverting Equation (2) to get

$$
\theta=\log \frac{\sigma}{a e^{b}+c e^{d}} .
$$

However, any arbitrary non-linear stiffness characteristic (compatible with device stiffness workspace) can be reproduced. This can be achieved still relying on system characterization and the knowledge of the indentation value $\delta$. This value can be retrieved in active touch modality using the IR sensor (value $h_{a}$ ), while in the passive mode this corresponds to the servo commanded displacement $h_{b}$. Without loss of generality, we report the case of power $F(\delta)$ function, i.e., $F(\delta)=\lambda \delta^{\beta}$. It is worth to note that any other function representing a nonlinear stiffness profile can be reproduced. We chose power functions since they are commonly used to describe the stiffness behavior of real specimens, see, e.g., [26], [28]. As in [26], [28], the motor position to reproduce the value of $\lambda$ (assuming the knowledge of the power exponent) can be determined as

$$
\lambda=\frac{F}{\delta^{\beta}}=\frac{\sigma(\theta) \delta}{\delta^{\beta}}=\frac{a e^{b \theta}+c e^{d \theta}}{\delta^{b-1}} .
$$




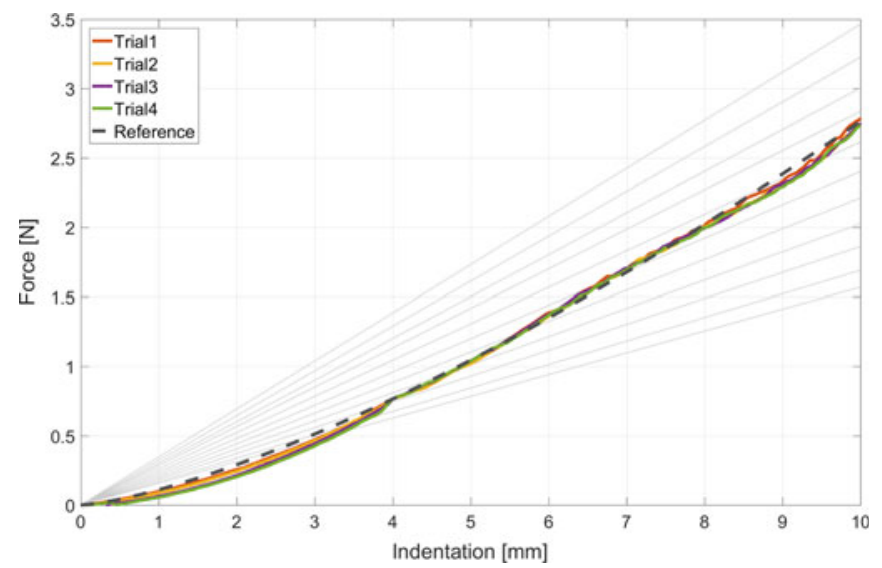

Fig. 6. Raw data for the rendered profiles. Dashed line is the reference curve, while the rendered ones are indicated as Trial i, where i refers to trial number. It is worth noticing a good repeatibility of system behaviour. In gray the characterization curves (Fig. 5).

After some algebra, we obtain

$$
\theta=\log \frac{\lambda \delta^{\beta-1}-q}{a e^{b}+c e^{d}} .
$$

Note that while interacting with the W-FYD, typical dynamic human mode for successful softness probing is enabled. This basically consists of pressing the finger along the normal to the surface, as widely discussed in literature (see, e.g., the exploratory procedures described in [32], or [18], where anisotropic or distortion effects in softness perception were ascribed to lateral force exertion).

To simulate also dynamic aspects related to softness rendering, we would basically need to retrieve the velocity of the interaction (i.e., $d h_{a} / d \delta$ and $d h_{p} / d \delta$ ). This value could be hence used to switch across different stiffness curves obtained in function of the indentation velocity, and the here reported stiffness rendering can be again employed. Finally, it is also worth to note that viscoelasticity of the fabric is negligible, as demonstrated in [26].

\subsection{Non-Linear Stiffness Rendering Evaluation and System Identification}

To complete the characterization of the system, it is important to test W-FYD performance in non-linear softness rendering. To tackle this issue, we need (i) to assess W-FYD performance in varying the stretching state of the fabric through the DC motors to reproduce a given stiffness profile (which is important for both active and passive exploration) and (ii) to retrieve the transfer function between the commanded inputs to the servo motor and the effective indentation generated by the device (which is important to determine the bandwidth of W-FYD response for passive touch interaction). It is worth noticing that point (i) is common to both active and passive touch, the only difference is the mean we use to get indentation information. For this reason, without loss of generality, we report the experiment we performed for the case of active exploration only.

\subsubsection{Non-Linear Stiffness Rendering Evaluation}

For this experiment, we used the support structure, indenter and testing machine already employed for the characterization and described in Section 3.1. Since we focus on the active mode only, we controlled the machine to generate a repeatable indentation step on the fabric of $10 \mathrm{~mm}$, with a speed of $3 \mathrm{~mm} / \mathrm{s}$. This value was chosen as a reasonable indentation velocity used by humans during unconstrained indentation experiments [20]. DC motors of W-FYD were hence controlled according to the control law reported in Equation (5) and relying on the measurements of the IR sensor. Force information was also recorded and both force and indentation information were synchronized via software.

In this experiment, we chose to mimic the power $F(\delta)$ function, $F(\delta)=\lambda \delta^{\beta}=0.11 \delta^{1.40}$, since it perfectly spans all the device stiffness workspace, see Fig. 6.

To perform a numerical analysis of the results, the Percentage Normalized Root Mean Square Error (PNRMSE) was computed as

$$
P N R M S E=100 \frac{\sqrt{\frac{\sum_{d=0}^{N}\left(f_{r}(i(d))-f_{t}(i(d))\right)^{2}}{N}}}{\max _{i} f_{t}(i(d))-\min _{i} f_{t}(i(d))},
$$

where $f_{r}(i)$ is the reference force profile, $f_{t}(i)$ is the simulated force profile, $i$ represent the indentation, $d$ represent the sample, $N$ is the total number of samples.

We considered the difference between the requested force and the obtained force given an indentation value for four different trials. We obtained a PNRMSE of 8.18 percent which is comparable with the results in [26].

\subsubsection{System Identification}

To determine the bandwidth of W-FYD response for passive touch interaction, we run an identification experiment to retrieve the transfer function between the commanded inputs to the servo motor and the effective indentation generated by the device. To achieve this goal, we used an active marker-based motion tracking system (PhaseSpace Motion Capture system, PhaseSpace Inc.) to measure the effective displacement of the lifting system. This system is highly accurate (the amount of static marker jitter is inferior than $0.5 \mathrm{~mm}$, usually $0.1 \mathrm{~mm}$ ). We placed two markers, one on the base and the other on the lifting mechanism to measure the relative displacement between them.

We used different sinusoidal profiles as position input to the servo motor, with a frequency range from 1 to $20 \mathrm{~Hz}$, with step of $0.2 \mathrm{~Hz}$. Each sinusoidal input was maintained for 50 complete waves for any frequency. For each input we computed the ratio between the amplitude of the main component of the output and the amplitude of the sinusoidal input in frequency domain. These coefficients were used to devise the Bode diagram of the transfer function.

From Fig. 7 it is possible to observe that the system exhibits an expected low pass behavior with cut frequency at $-3 \mathrm{~dB}$ in $3.04 \mathrm{~Hz}$ and a cut frequency at $-6 \mathrm{~dB}$ in $4.36 \mathrm{~Hz}$. These results are good since in preliminary experiments we observed that the value of $3 \mathrm{~Hz}$ can be regarded as a cautelative upper bound for typical indentation frequency used by humans during the active interaction with the W-FYD. 


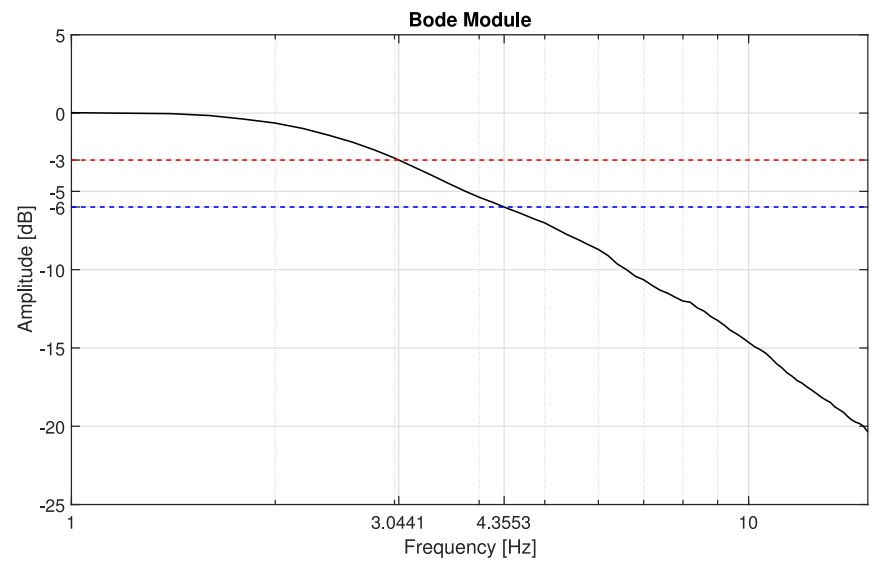

Fig. 7. Bode diagram of the estimated Transfer Function. Dotted red line defines the $-3 \mathrm{~dB}$ bandwidth, while the blue dotted line indicates the $-6 \mathrm{~dB}$ badwidth.

\section{Psychophysical Experiments: Methods}

To test the effectiveness of W-FYD in conveying reliable softness and sliding cues and assess whether the device weight can represent a factor that could affect the elicited tactile perception in users, we performed a thorough psychophysical characterization of the system in terms of Just Noticeable Difference (JND) and Weber fraction. Both active and passive modes were tested. A suitable mechanical support was designed to compensate the effect of the weight of the device, as depicted in Fig. 8 and described later in the text.

For softness discrimination, we used the method of constant stimuli and performed two types of experiments. In the first experiment, hereinafter referred to as Device Linear Softness Discrimination, W-FYD was commanded to provide different levels of linear stiffness (i.e., corresponding to different motor positions), and both the reference and comparison stimuli were artificially reproduced. In the second experiment, hereinafter referred to Device Non-linear Softness Discrimination the same experimental conditions used for the linear case were employed but in this case motors were controlled to reproduce a power force-indentation profile, as described in the previous Section, and no weight compensation implemented.

To characterize the performance in discriminating the entity of the sliding stimuli, we performed similar experiments with the method of constant stimuli, where the device was controlled to induce right and left displacement on the user's finger-pad, controlling the motor to coherently rotate clockwise and counter-clockwise, respectively. In this case, we considered all the configurations, i.e., with and without weight compensation.

Finally, we report on Relative Recognition Task, where participants were asked to probe and sort for softness three different non-linear force-indentation profile for both active and passive touch. The results of a subjective quantitative evaluation by users based on a Likert-type scale are also discussed, with questions related on W-FYD wearability and effectiveness in haptic multi-cue delivery.

\subsection{Device Linear Softness Discrimination}

By means of psychophysical experiments, we evaluated the capacity of human participants in discriminating the

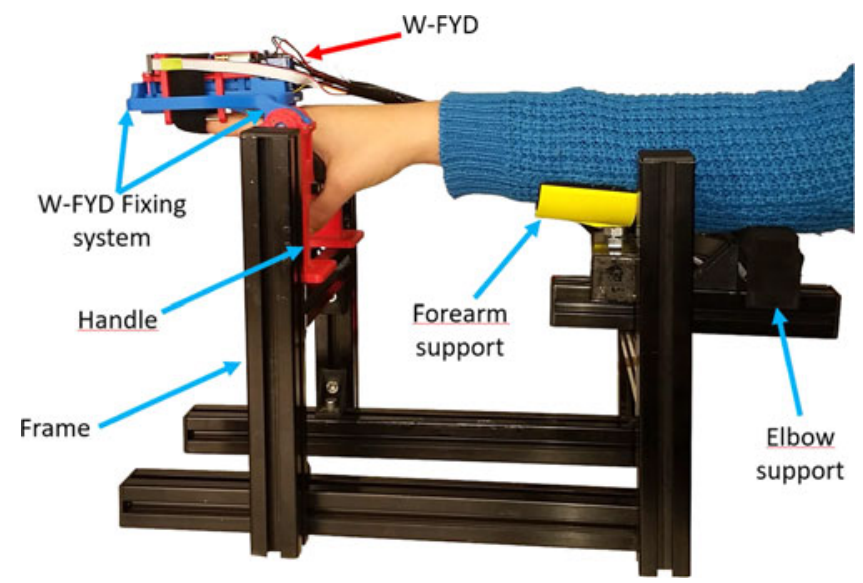

Fig. 8. Support structure used during the psychophysical experiment.

different levels of linear softness rendered by the device, which correspond to fixed motor positions. We used the method of constant stimuli [33]. In a forced-choice task, we presented participants with two stimuli of different compliance and asked them to report which of the two was felt as less soft. The two operating modes of the device were used, the passive- and the active-touch mode, either with the weight of the device supported by a custom made structure (Fig. 8) or with the weight completely supported by the participant. The custom made structure showed in Fig. 8 was composed of a frame to which an elbow support and a forearm support are fixed. They are used to avoid fatigue in the participant and to help keeping the arm in the right position, and a handle kept in the wrist of the participants. To the handle we placed a removable structure to which the W-FYD can be fixed to support its weight. Four experimental condition were used: Active touch with weight support (labeled as Active Weight-support plus, or $A W p$, in the manuscript), active touch without weight support (Active Weightsupport minus or $A W m$ ), passive touch with weight support (Passive Weight-support plus or PWp), passive touch without weight support (Passive Weight-support minus or PWm). Each condition was tested in a separate experimental block.

The two Weight-support plus conditions were included to evaluate whether the weight of the device affects the perceived softness.

\subsubsection{Participants}

Twelve right-handed healthy participants (3 Female, Age mean \pm SD: $26.83 \pm 3.45)$ gave their informed consent to participate in the experiment. Before each experimental session, the experimenter verified that participant's finger-pad was free of calluses. No physical neither mental disorder was present that could have affected the experimental outcome. The experimental procedure was approved by the Ethical Committee of the University of Pisa

\subsubsection{Experimental Setup}

Participants were comfortably seated on an office chair, with their arm on the support structure. The elbow and the forearm were lying on the respective support structures. The height of the chair was regulated such that the forearm was kept parallel to the transverse plane of the participant. 
Participants were asked to keep their hand on the handle in both Weight-support plus and Weight-support minus conditions, the difference between the two being in the fixing of the W-FYD on the supporting structure (in blue in Fig. 8), which completely sustains the weight of the device. The index finger was kept inside the W-FYD with the finger-pad in correspondence with the fabric stripe and the finger nail below the IR sensor of the device.

In all the experiments, auditory pink noise was delivered to the participants via headphones in order to mask any sound from the device. Participants were blind-folded through the usage of a pair of goggles with obscured lenses, to prevent the view of the system. Within each trial, a reference and a comparison stimulus were presented to the participant in a pseudo-random order. After the second stimulus was off, the participant reported which stimulus in the pair was felt as less soft. Response time was unconstrained. Participants could not request any repetition of the presented stimuli. They were asked to indent the elastic fabric by moving the second and the third phalanges of the index finger (note that the device blocked the metacarpophalangeal joint, see Fig. 3d). Five discrete and equally spaced stimuli were used, the same for all conditions. The stimuli values were expressed in terms of stiffness coefficient (referring to the device characterization) and were equal to $0.21,0.235,0.26,0.285,0.31 \mathrm{~N} / \mathrm{mm}$ (Reference Stimulus $0.26 \mathrm{~N} / \mathrm{mm}$ ). The stimuli duration was not fixed, the participant decided to move to the second stimulus, while inter-stimulus interval was $1.5 \mathrm{~s}$.

Four experimental condition ( $A W p, A W m, P W p, A W m)$ were tested in four experimental blocks. The order of the four blocks was counterbalanced across participants. Each block consisted of 100 trials, presented in a pseudo-random order. We used a different random sequence of the stimuli in the four blocks. Answers of the participants were recorded with a computer keyboard and saved for future analysis. The sequence of the stimuli and the apparatus were controlled by a custom-made C software.

\subsubsection{Data Analysis}

By means of a Generalized Linear Mixed Model (GLMM), we tested whether the compliace of the fabric, $k$, and the modatility of exploration (active and passive, with and without weight support) affected the perceived softenss. GLMMs accounts for the effect of the experimental variables and for the variability between participants by means of fixed- and random-effect parameters, respectively [34]. We used a model of the form

$$
\begin{aligned}
\Phi^{-1}[P(Y=1)]= & \beta_{0}+u_{0}+\beta_{1} k+u_{1} k+\beta_{2}\left(A W_{m} k\right) \\
& +\beta_{3}\left(P W_{p} k\right)+\beta_{4}\left(P W_{m} k\right),
\end{aligned}
$$

where $\Phi^{-1}$ is the probit link function, $P(Y=1)$ is the probability of perceiving the comparison stimulus as less soft than the reference, $\beta_{*}$ and $u_{*}$ are the fixed- and random-effect parameters, respectively. We used the three dummy vairables $A W m, P W p$ and $P W m$ to account for all the experimental conditions (with $A W p$ being baseline in the model). Next, we estimated the Just Noticeable Differences (JND) in the four experimental conditions as explained in [34].

\subsection{Device Non-Linear Softness Discrimination}

We evaluated the capacity of human participants to discriminate the stimuli in a pool of non-linear softness profiles. In a forced-choice task, we presented participants with two stimuli of different non-linear compliance and asked them to report which of the two was felt as less soft. Both the passive and the active modes of the device were tested. In this case only the condition without weight support was used (Experiment 1 already excluded possible confounds due to device weight, see Section Results). This resulted in two experimental condition: Active touch without weight support $(A W m)$ and passive touch without weight support $(P W m)$. Each condition was tested in a separate experimental block.

\subsubsection{Participants}

Twelve right-handed healthy participants (6 Female, Age mean \pm SD: $27.00 \pm 4.36$ ) gave their informed consent to participate in the experiment. No one had any physical limitation which would have affected the experimental outcomes. Their finger-pads were free of calluses. The experimental procedure was approved by the Ethical Committee of the University of Pisa

\subsubsection{Experimental Setup}

The setup was similar to the one described in Section 4.1.2. In this case only the configuration without the weight support was performed. To do this, we removed the W-FYD from the support structure.

As before, participants were completely insulated from all the possible disturbances, both acoustic and visual. Within each trial, a reference and a comparison stimulus were presented to the participant in a pseudo-random order. After the second stimulus was off, the participant reported which stimulus in the pair was felt as less soft. The participant was able to choose when to move to the next stimuli, without any time constraint. They were not allowed to request any repetition of the presented stimuli and they were asked to perform the investigation of the softness moving only the second and the third phalanges.

We used a different random sequence of the stimuli in the two blocks. The order of the two blocks was counterbalanced across participants. Five discrete and equally spaced stimuli were used for all the combinations. In this case the chosen stimulus was a non-linear softness profile, particularly a power function as for the non-linear softness rendering evaluation experiment (see Section 3.3). The reference stimuli was chosen exactly as the one of the experiment in Section $3.3 F(\delta)=\lambda \delta^{\beta}=0.11 \delta^{1.40}$. The stimuli considered for the JND was this time the variation of $\lambda$. The extreme stimuli were chosen via a preliminary study as the signals that were almost always perceived less or more stiff than the reference one. The chosen values of $\lambda$ were $0.09,0.10$, $0.11,0.12,0.13 \mathrm{~N} / \mathrm{mm}$ (Reference Stimulus $0.11 \mathrm{~N} / \mathrm{mm}$ as previously described), and are showed in Fig. 9.

Each block consisted of 100 trials, presented in a pseudorandomized order. Answers of the participants were recorded with a computer and saved on a file for future analysis. Even this experiments were automatically managed by a custom C-written software. The obtained results 


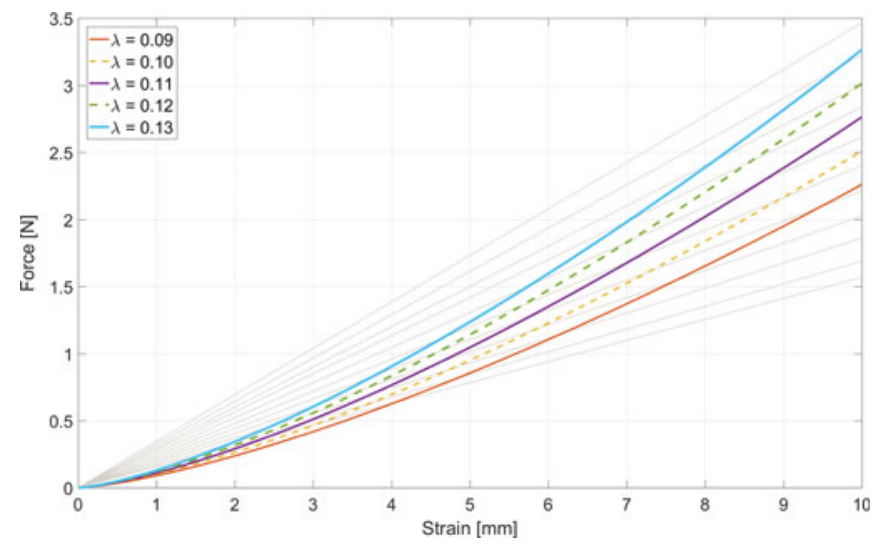

Fig. 9. In this figure all the stiffness profiles used in the Non-linear Softness Discrimination esperiment are reported. In the backgroung (in gray) we plotted the characterization curves (Fig. 5).

were then analyzed to obtain the JND of the participant perception of the rendered stiffness coefficient.

\subsubsection{Data Analysis}

We tested whether the non-linear compliance of the fabric, characterized by the parameter $\lambda$ in Equation (4), affected the perceived softness in active and passive exploration. We fit the data using a GLMM similar to the one in Equation (7). This time $\lambda$ was the continuous predictor. We estimated the JND in active and passive condition.

\subsection{Tangential Displacement Discrimination}

In a third experiment we evaluated the capacity of human participants to discriminate the length of tangential displacement of the fabric sliding over the finger-pad. In the previous conference paper [22], we demonstrated that subjects were able to correctly discriminate the direction of sliding. Here, in a forced-choice task, we presented participants with two stimuli of different displacement length and asked them to report which of the two was felt as larger. The two possible direction of movement, fabric sliding to the right (DC motors clockwise rotation) and to the left (DC motors counterclockwise rotation), were tested, while their starting position was set to zero (with respect to Fig. 5). Also in this experiment both conditions, with the weight of the device supported by a custom made structure (see Fig. 8) and with the weight completely supported by the participant, were used. This resulted in four experimental condition: slide to right with weight support (labelled as $R W p$ in the manuscript), slide to right without weight support $(R W m)$, slide to left with weight support $(L W p)$, slide to left without weight support $(L W m)$. Each condition was tested in a separate experimental block.

\subsubsection{Participants}

Twelve right-handed healthy participants (5 Female, Age mean \pm SD: $26.83 \pm 3.38$ ) gave their informed consent to participate in the experiment. No one had any physical limitation which would have affected the experimental outcomes. Their finger-pads were free of calluses. The experimental procedure was approved by the Ethical Committee of the University of Pisa

\subsubsection{Experimental Setup}

The setup was the same as the one described in Section 4.1.2.

During the experiments, subjects wore headphones with pink noise to prevent auditory cues. In addition they were asked to wear a pair of goggles with obscured lenses to prevent the view of the system. As in all the other JND experiments we performed, within each trial, a reference and a comparison stimulus were presented to the participant once in a pseudo-random order. After the second stimulus was off, the participant reported which stimulus in the pair was perceived to produce a larger sliding. Subjects were not allowed to request any repetition of the presented stimuli. We used a different random sequence of the stimuli in the four blocks. The order of the four blocks was counterbalanced across participants.

Five discrete and equally spaced stimuli were used for all the combinations. The extreme stimuli were chosen via a preliminary study as the signals that were almost always perceived less or more wide than the reference one. The stimuli values were expressed in terms of motor angles commanded to both motors as 30, 60, 90, 120, 150 degree (Reference Stimulus 90 degree), corresponding to $3.14,6.28,9.42,12.56,15.70 \mathrm{~mm}$ (with reference $6.42 \mathrm{~mm}$ ) fabric sliding. Each block consisted of 100 trials, presented in a pseudo-randomized order. Answers of the participants were recorded with a computer and saved on a file for future analysis. The experiments were automatically managed by a custom C-written software. The obtained results were then analyzed to obtain the JND of the subject perception of the lateral sliding in the four different conditions.

\subsubsection{Data Analysis}

We tested whether participants were able to discriminate the length of the tangential displacement of the fabric, with and without device weight support. As in previous experiments, we fit the data using a GLMM. Here the displacement length was the continuous predictor. We estimated the JND for leftward and rightward motion, with and without device weight support.

\subsection{Subjective Quantitative Evaluation}

In the last experiment, we asked participants to perform a relative cognitive tasks and underwent through a subjective quantitative evaluation of the device.

In the first part, participants were asked to touch three rendered non-linear stiffness profiles, and to rank them in ascending order on the basis of the perceived stiffness. In the second part, a series of sliding movement were presented to the participants and they were asked to recognize the direction of the sliding movement (giving the answer in terms of finger sliding direction).

In both the parts, we considered active touch interaction with and without the use of the weight support. We chose only the active mode since our goal was to test the device in a dynamic task, where users' fingers and hands were free to move. After performing the two parts, participants underwent the subjective quantitative evaluation procedure based on a seven point Likert-type scale. 


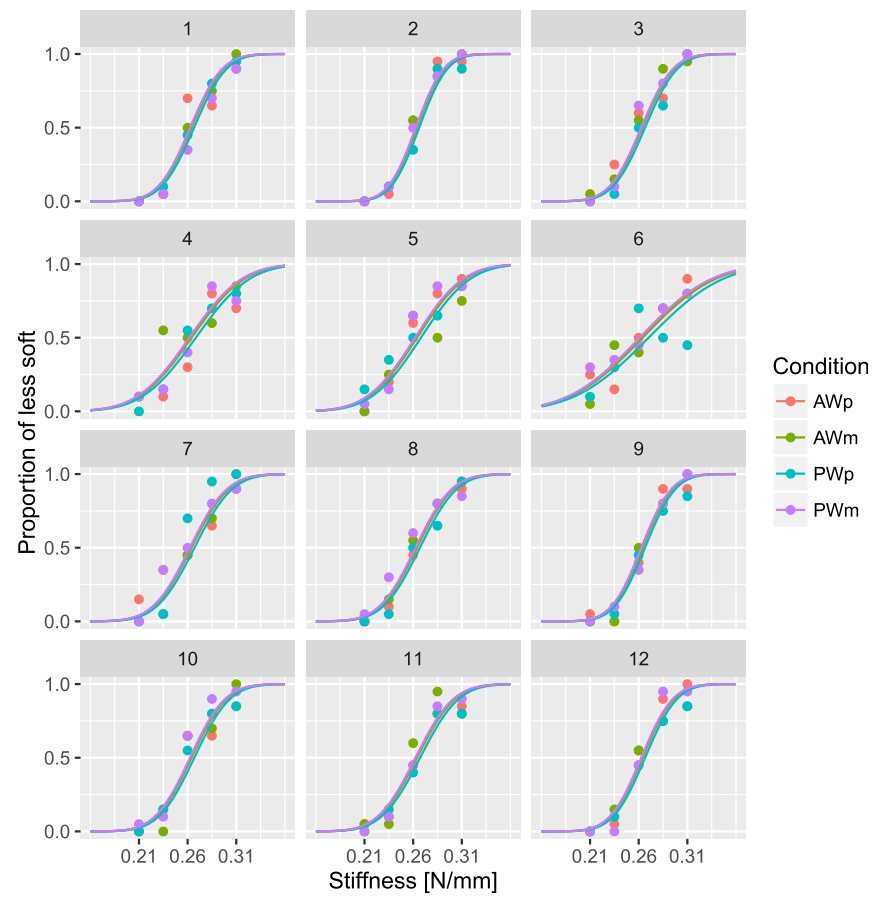

Fig. 10. Results of linear softness discrimination task in the four experimental conditions. Each box shows data and GLMM fit for a different participant.

\subsubsection{Participants}

Twelve right-handed healthy participants (4 Female, Age mean \pm SD: $27.75 \pm 3.88$ ) gave their informed consent to participate in the experiment. No one had any physical or mental limitation which would have affected the experimental outcomes. Their finger-pads were free of calluses. The experimental procedure was approved by the Ethical Committee of the University of Pisa.

\subsubsection{Experimental Task}

The experimental setup was similar to the one used in the previous experiments. In this case, since all the experiment was performed without support structure, the W-FYD fixing system was removed.

In the first part of the experiment, we focused on the softness ordering part, considering three different non-linear softness. Coherently with the rest of the experiments, we used three power functions $F(\delta)=\lambda \delta^{1.40}$, more precisely the lower bound $(\lambda 0.09)$, the reference value $(\lambda 0.11)$ and the higher bound ( $\lambda 0.13)$, used in the experiment for the nonlinear softness JND (see Section 4.2.2). The three values were presented six times in random order and the participants were asked to probe and sort them in terms of softness. Participants did not have time limitation and they had the possibility to ask the repetition of a single stimulus or of all the three stimuli in the same order before giving the answer. To analyze these data, we summed all the results for all the subjects in a confusion matrix reporting the perceived softness versus the rendered one.

For the second experiment, 30 tangential sliding stimuli $(60 \mathrm{~mm})$ were presented in a pseudo-random order to the participants. 15 of them were to the right and 15 to the left. Every stimuli was presented once and the subject had to answer immediately giving the sliding direction he perceived. At the end of the experiments, participants underwent a subjective quantitative evaluation using a seven point Likert-type scale, taking inspiration from [11] and reported in Table 2, where a value of 1 means totally disagree and 7 totally agree.

\section{PSYChOPHYSICAL EXPERIMENTS: ResULTS}

\subsection{Device Linear Softness Discrimination}

By means of psychophysical experiments, we evaluated the capacity of human participants in discriminating the difference levels of softness redered by the device. We tested four experimental condition: Active touch with weight support $(A W p)$, active touch without weight support $(A W m)$, passive touch with weight support $(P W p)$, and passive touch without weight support $(P W m)$.

We modeled the data with Equation (7). The slope of the model in baseline condition $(A W p)$ was significantly different from zero $\left(\beta_{1}=33.7, p<0.001\right)$, meaning that participants were able to discriminate the stimuli. In conditions $A W m, P W p$ and $P W m$ the slope of the model was non significantly different from the baseline condition. Accordingly, the confidence intervals (CIs) of the JND in the four conditions were largely overlapping. The JND was equal to $0.0200 \mathrm{~N} / \mathrm{mm}$ in $A W p$ (95 percent CI ranging from 0.0174 to $0.0231 \mathrm{~N} / \mathrm{mm}$ ), $0.0199 \mathrm{~N} / \mathrm{mm}$ in $A W m$ (95 percent CI ranging from 0.0175 to $0.0231 \mathrm{~N} / \mathrm{mm}), 0.0202 \mathrm{~N} / \mathrm{mm}$ in $P W p$ (95 percent CI ranging from 0.0176 to $0.0234 \mathrm{~N} / \mathrm{mm}$ ), and $0.0198 \mathrm{~N} / \mathrm{mm}$ in $P W m$ (95 percent CI ranging from 0.0173 to $0.0231 \mathrm{~N} / \mathrm{mm}$ ). Average Weber Fraction, computed as the ratio between the JND and the reference stimulus, was approximately equal to 8 percent.

In summary, participants were able to discriminate the different softness values. Neither the weight support nor the active/passive exploration affected the response significantly (see Fig. 10). The similarity between active and passive condition is in accordance with [20]. Average Weber Fraction was slightly smaller compared with values previously reported in the literature with real objects, which ranged from 13 to 15 percent [19]. The difference in materials (silicones in previous studies) and in the stifness range (reference value around $9 \mathrm{~N} / \mathrm{mm}$ in [19]) may possibly explain these differences.

\subsection{Device Non-Linear Softness Discrimination}

Here we evaluated the capacity of human participants in discriminating the non-linear softness profile reported in Section 3. We tested two experimental conditions, active and passive touch (labeled as $A W m$ and $P W m$, respectively) without weight support. We made this choice since results from Section 5.1 show no difference between weight and no-weight support

We modeled the data using GLMM with different values of $\lambda$ as continuous predictors. The parameter $\lambda$, as defined in Equation (4), characterizes the non-linear compliance of the different stimuli. The slope of the model in baseline condition $(A W m)$ was significantly different from zero $\left(\beta_{1}=90\right.$, $p<0.001)$, meaning that participants were able to discriminate different values of $\lambda$. In condition $P W m$ the slope of the model was non significantly different from the baseline condition. Accordingly, confidence intervals of the two JNDs were largely overlapping. The JND was equal to 


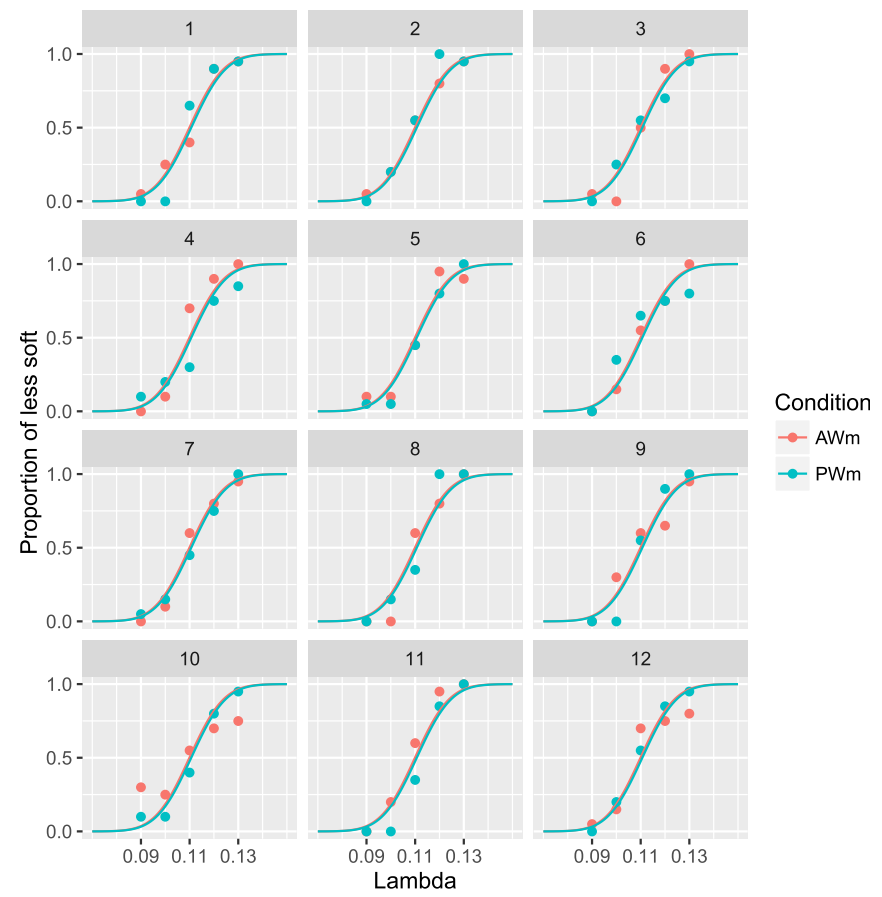

Fig. 11. Results of nonlinear softness discrimination task in the two experimental conditions. Each box show data and GLMM fit for a different participant.

$0.0075 \mathrm{~N} / \mathrm{mm}$ in $A W m$ (95 percent CI ranging from 0.0069 to $0.0079 \mathrm{~N} / \mathrm{mm}$ ) and $0.0075 \mathrm{~N} / \mathrm{mm}$ in $\mathrm{PWm}$ (95 percent CI ranging from 0.0070 to $0.0080 \mathrm{~N} / \mathrm{mm}$ ). Averege Weber fraction was equal to approximately 7 percent.

In summary, participants were able to discriminate the different values of non-linear softness. The active/passive exploration did not affect the response significantly (see Fig. 11).

\subsection{Tangential Displacement Discrimination}

We evaluated the capacity of human participants to discriminate the tangential displacement of the fabric. The fabric sliding over participant's finger, either leftward or rightward. We tested four experimental conditions: leftward motion with weight support $(L W p)$, leftward motion without weight support $(L W m)$, rightward motion with weight support $(R W p)$, and rightward motion without weight support $(R W m)$.

We modelled the data using GLMM. The slope of the model in baseline condition $(R W p)$ was significantly different from zero $\left(\beta_{1}=0.44, p<0.001\right)$, meaning that participants were able to discriminate the displacement length. In conditions $R W m, L W p$ and $L W m$ the slope of the model was non significantly different from the baseline condition. Accordingly, the CIs of the JND in the four conditions were largely overlapping. The JND was equal to $1.53 \mathrm{~mm}$ in $R W p$ (95 percent CI ranging from 1.39 to $1.66 \mathrm{~mm}$ ), $1.54 \mathrm{~mm}$ in $R W m$ (95 percent CI ranging from 1.40 to $1.69 \mathrm{~mm}$ ), $1.56 \mathrm{~mm}$ in $L W p$ (95 percent CI ranging from 1.43 to $1.70 \mathrm{~mm}$ ), and $1.55 \mathrm{~mm}$ in $P W m$ (95 percent CI ranging from 1.42 to $1.69 \mathrm{~mm}$ ). Average Weber fraction was approximately equal to 17 percent, which is close to value reported in [35] with a hard surface $($ Weber fraction $=19 \%$ ).

In summary, participants were able to discriminate the different displacement lengths. Neither the weight support
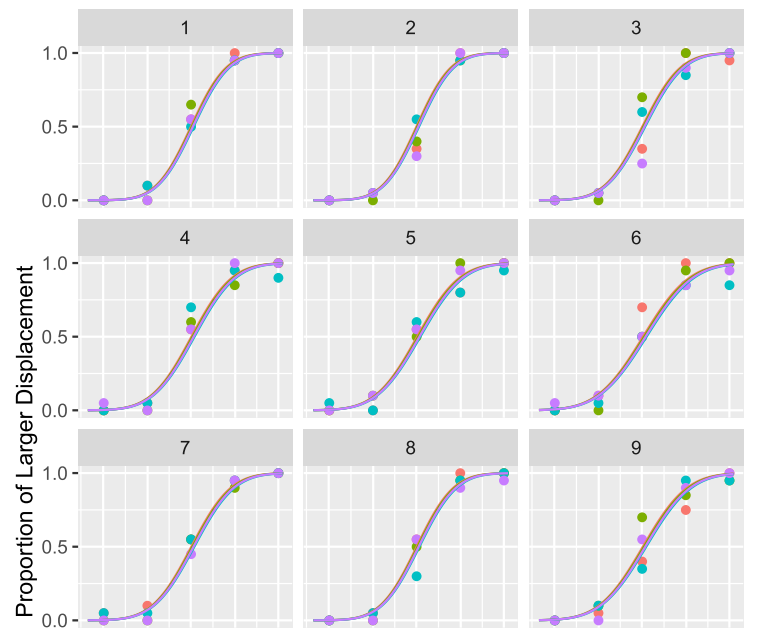

Condition

$\rightarrow$ RWp

9

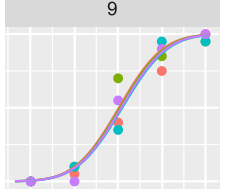

$\rightarrow$ RWm

$\rightarrow$ LWp

$\rightarrow \mathrm{LWm}$
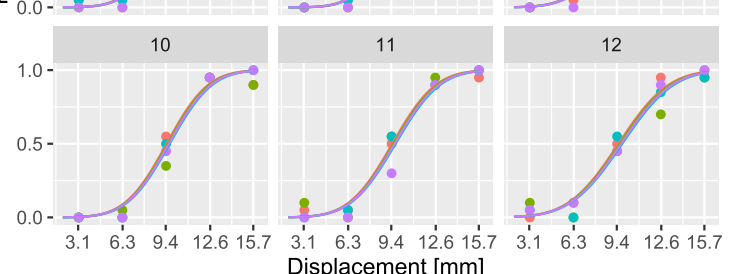

Displacement [mm]

Fig. 12. Results of displacement discrimination task in the four experimental conditions. Each box shows data and GLMM fit for a different participant.

nor the motion direction affected the response significantly (see Fig. 12).

\subsection{Subjective Quantitative Evaluation}

Results of the first part of the experiment, i.e., softness ranking task, are shown in Table 1, where perceived softness is reported versus artificially reproduced softness, with the relative accuracy in discrimination for each softness value. The average recognition rate is $87.97 \% \pm 5.30 \%$ (mean \pm SD, chance level of 33.34 percent), indicating that the device is able to render softness levels that are well recognizable by the participants.

For the second part of the experiment, i.e., the sliding direction recognition, the success rate was 99.2 percent (100 percent for the left stimuli and 98.3 percent for the right ones), indicating that direction of sliding stimuli is highly distinguishable by participants.

After the experiment, participants were asked to answer 11 questions using a Likert-type seven-point scale. In Table 2 the participants answers are presented in terms of mean value and standard deviation between all the participants, where a value of 1 means totally disagree and 7 totally agree.

Results show that participants perceived W-FYD as easy to wear and comfortable $(\mathrm{Q} 1, \mathrm{Q} 2, \mathrm{Q} 8, \mathrm{Q} 9)$. Furthermore, they were able to correctly interpret the stimuli provided by the device for both softness rendering and sliding $(Q 5, Q 6)$ and

TABLE 1

Confusion Matrix for Relative Recognition Task

\begin{tabular}{lcccc}
\hline & SH1 & SH2 & SH3 & Relative Accuracy \\
\hline SS1 & 66 & 6 & 0 & $91.7 \%$ \\
SS2 & 6 & 59 & 7 & $81.9 \%$ \\
SS3 & 0 & 7 & 65 & $90.3 \%$ \\
\hline
\end{tabular}


TABLE 2

These Statements, Presented in Random Order, were Rated by Subjects Using a Seven-Point Likert Scale (1: Strongly Disagree, 7: Strongly Agree)

\begin{tabular}{|c|c|c|c|}
\hline & Questions & Mean & Std. Dev. \\
\hline Q1 & $\begin{array}{l}\text { It was easy to wear and use the } \\
\text { W-FYD device }\end{array}$ & 6.50 & 0.65 \\
\hline Q2 & $\begin{array}{l}\text { It was feeling uncomfortable while } \\
\text { using the W-FYD }\end{array}$ & 2.75 & 1.59 \\
\hline Q3 & $\begin{array}{l}\text { I was well isolated from external } \\
\text { noises }\end{array}$ & 6.83 & 0.37 \\
\hline Q4 & $\begin{array}{l}\text { I was able to hear the sounds made } \\
\text { by the actuators of the W-FYD }\end{array}$ & 1.67 & 0.94 \\
\hline Q5 & $\begin{array}{l}\text { The stimuli provided by the } \\
\text { cutaneous device were enough to } \\
\text { discriminate the softness }\end{array}$ & 6.00 & 0.82 \\
\hline Q6 & $\begin{array}{l}\text { The stimuli provided by the } \\
\text { cutaneous device were enough to } \\
\text { discriminate the direction of sliding }\end{array}$ & 6.92 & 0.28 \\
\hline Q7 & $\begin{array}{l}\text { At the end of the experiment I felt } \\
\text { tired }\end{array}$ & 1.08 & 0.28 \\
\hline Q8 & $\begin{array}{l}\text { It was easy to move my hand and } \\
\text { fingers while wearing the cutaneous } \\
\text { device }\end{array}$ & 6.00 & 1.00 \\
\hline Q9 & I felt hampered by the W-FYD & 2.50 & 1.50 \\
\hline Q10 & $\begin{array}{l}\text { The stimuli provided by the } \\
\text { cutaneous device on the fingertip } \\
\text { felt strange }\end{array}$ & 2.08 & 1.11 \\
\hline Q11 & $\begin{array}{l}\text { I felt the stimuli provided by the } \\
\text { cutaneous device only on the } \\
\text { fingertip }\end{array}$ & 6.75 & 0.43 \\
\hline
\end{tabular}

Means and standard deviations for each participant are reported.

perceived them as natural (Q10). These stimuli were experienced only at fingertip level, which is an additional proof of device wearability (Q11). Q3 and Q4 show that the experimental conditions were sound, and Q7 provides indication that the load on the subjects from the experiment was not excessive.

\section{Applications in Tactile Augmented Reality AND Open SuRgery Training}

As previously mentioned in the Introduction, leveraging upon its wearability and deformability of the fabric, the architecture of the W-FYD can open fascinating perspectives in many application fields for human-machine interaction, e.g., surgical training, paving the path towards a new paradigm named tactile augmented reality.

Surgeons strongly rely on tactile and kinesthetic feedback to differentiate tissues and other hidden structures. Because of this, the use of haptic devices in medical applications has been previously proposed in different forms. Grounded haptic systems are usually adopted for palpation as in [36] or in surgical training systems for needle insertion [37]. Wearable solutions are mainly thought for Virtual Reality (for an exhaustive review on these systems please refer to [38]). However, W-FYD features are unique. First it is able to deliver multiple cues, furthermore the usage of a fabric as interaction surface allows to superimpose tactile effects on real objects, without impairing cutaneous sensations, within the paradigm of tactile augmented reality.
In this Section, we summarize the results of an interesting implementation of the this paradigm [29]. More specifically, we integrated the W-FYD within an augmented physical simulator endowed with high-fidelity physical models of human organs and augmented reality (AR) functionalities for open-surgery cholecystectomy simulation. The goal was to take advantage from the high wearability of the W-FYD, which enables an easy integration with human body, and the usage of the fabric as interaction surface with user's finger-pad, which provides minimal cutaneous impairment during haptic probing of the anatomical structures of AR simulator. Indeed, we were able to add additional tactile cues for an increased realism, superimposing artery pulse effect to artificial organs without compromising the sensation derived from artery palpation. It is worth to observe that pulse feedback during palpation represents an essential requirement for the correct identification of the cystic and right hepatic artery.

For this work, W-FYD was customized, removing the two pins from the base, thus having the fabric directly wrapped around user's finger-pad. Only the passive mode was employed to deliver pressure cues to the finger-pad to simulate artery pulse, without considering stiffness rendering. Artery pulse was reproduced commanding a saw-tooth height variation to the lifting mechanism and varying the length of the pulse period to render different heart rates. A bang-bang control model activated the pulse feedback based on the fulfillment of two criteria: proximity condition of the finger w.r.t the artery (relying on the finger position retrieved through a sensorization via a 6DOF EM Aurora Sensor and a calibration procedure) and the interaction force with the artery (measured through a thin load cell sewn on the external part of the fabric). Activation thresholds were identified in preliminary experiments with humans. We performed a subjective quantitative evaluation of the integrated system with ten surgeons who were required to use the AR simulator with the W-FYD to recognize the cystic or right hepatic artery. Results showed an overall agreement on the realism of the pulse feedback and the effectiveness and usefulness of the integration of W-FYD within the simulator (further details can be found in [29]).

Despite such promising results, in some of the tests, surgeons identified the effect of weight as a potential issue to consider for real applications in RMIS. For these reasons, we will work on further reducing the dimensions of the device, as discussed in Section 7.

\section{Conclusions AND Future WORKS}

In this paper we have presented the W-FYD (WearableFabric Yielding Display, see Fig. 1a), a wearable fabric based device, which enables, for the first time, to convey controllable softness information to the user's finger-pad, allowing both active and passive haptic exploration [20]. Furthermore, stimuli related to tangential sliding can be also delivered. We have reported a thorough characterization of the system capabilities and performance, together with an exhaustive set of experiments with humans, including the estimation of W-FYD JNDs for all the types of stimuli it is able to convey. Results show that active and passive exploration seems to not affect participants' performance in 
softness discrimination, and such a result is coherent with [20]. Furthermore, the weight of the device does not impair users' capabilities in softness and tangential displacement recognition. These outcomes have also important implications for the study of human touch. For example, the good scores exhibited by participants in discriminating softness further sustain the importance of cutaneous elicitation as a fundamental cue for conveying softness information, as demonstrated also in [20], [39], [40]. Furthermore, the weight of the device appears to not have a crucial role in perceptual outcomes, as it is also confirmed by the subjective quantitative evaluation, where system wearability was perceived as high. The latter point further motivates a wearable approach in haptics for stimulus delivery and human studies. Finally, we also report on the integration of the W-FYD in AR simulator for surgical training, showing promising result for a tactile augmented framework relying on wearable haptics.

Conclusions that can be drawn are that W-FYD can be a viable solution for wearable haptic multi-cue delivery, with interesting perspectives in a wide range of fields, including but not limited to virtual and augmented reality, entertainment culture, tele-robotics and human robot interaction. Future works will aim at further pushing forward the wearability and miniaturization of the system, e.g., for possible multi-digit implementation. At the same time, different types of fabrics will be tested to increase device stiffness workspace.

\section{ACKNOWLEDGMENTS}

This work is supported in part by the EU H2020 project "SoftPro: Synergy-based Open-source Foundations and Technologies for Prosthetics and RehabilitatiOn" (H2020ICT-688857), the Advanced Grant SoftHands "A Theory of Soft Synergies for a New Generation of Artificial Hands" no. ERC-291166, and by the EU FP7 project (no. 601165), "WEARable HAPtics for Humans and Robots (WEARHAP)". The authors would like to thank Alessandro Serio and Mattia Poggiani for their valuable advice, and Federica Barontini and Davide Doria for their support in the experimental sessions.

\section{REFERENCES}

[1] J. Lieberman and C. Breazeal, "TIKL: Development of a wearable vibrotactile feedback suit for improved human motor learning," IEEE Trans. Robot., vol. 23, no. 5, pp. 919-926, Oct. 2007.

[2] R. Traylor and H. Z. Tan, "Development of a wearable haptic display for situation awareness in altered-gravity environment: Some initial findings," in Proc. 10th Symp. Haptic Interfaces Virtual Environment Teleoperator Syst., 2002, pp. 159-164.

[3] D. Leonardis, M. Solazzi, I. Bortone, and A. Frisoli, "A 3-RSR haptic wearable device for rendering fingertip contact forces," IEEE Trans. Haptics, to be published. doi: 10.1109/TOH.2016.2640291.

[4] M. Bianchi, et al., "Design and preliminary affective characterization of a novel fabric-based tactile display," in Proc. IEEE Haptics Symp., 2014, pp. 591-596.

[5] C. Pacchierotti, F. Chinello, M. Malvezzi, L. Meli, and D. Prattichizzo, "Two finger grasping simulation with cutaneous and kinesthetic force feedback," in Haptics: Perception, Devices, Mobility, and Communication. Berlin, Germany: Springer, 2012, pp. 373-382.

[6] G.-H. Yang, K.-U. Kyung, M. A. Srinivasan, and D.-S. Kwon, "Quantitative tactile display device with pin-array type tactile feedback and thermal feedback," in Proc. IEEE Int. Conf. Robot. Autom., 2006, pp. 3917-3922.
[7] K. J. Kuchenbecker, D. Ferguson, M. Kutzer, M. Moses, and A. M. Okamura, "The touch thimble: Providing fingertip contact feedback during point-force haptic interaction," in Proc. Symp. Haptic Interfaces Virtual Environment Teleoperator Syst., 2008, pp. 239-246.

[8] S. Casini, M. Morvidoni, M. Bianchi, M. G. Catalano, G. Grioli, and A. Bicchi, "Design and realization of the CUFF - clenching upper-limb force feedback wearable device for distributed Mechano-tactile stimulation of normal and tangential skin forces," in Proc. IEEE/RSJ Int. Conf. Intell. Robots Syst., 28 Sep.-2 Oct. 2015, pp. 1186-1193.

[9] F. Chinello, C. Pacchierotti, N. G. Tsagarakis, and D. Prattichizzo, "Design of a wearable skin stretch cutaneous device for the upper limb," in Proc. IEEE Haptics Symp., 2016, pp. 14-20.

[10] H. Kim, C. Seo, J. Lee, J. Ryu, S. Yu, and S. Lee, "Vibrotactile display for driving safety information," in Proc. IEEE Intell. Transp. Syst. Conf., 2006, pp. 573-577.

[11] D. Prattichizzo, F. Chinello, C. Pacchierotti, and M. Malvezzi, "Towards wearability in fingertip haptics: A 3-DoF wearable device for cutaneous force feedback," IEEE Trans. Haptics, vol. 6, no. 4, pp. 506-516, Oct.-Dec. 2013.

[12] B. T. Gleeson, S. K. Horschel, and W. R. Provancher, "Design of a fingertip-mounted tactile display with tangential skin displacement feedback," IEEE Trans. Haptics, vol. 3, no. 4, pp. 297-301, Oct.-Dec. 2010.

[13] K. Minamizawa, S. Fukamachi, H. Kajimoto, N. Kawakami, and S. Tachi, "Gravity grabber: Wearable haptic display to present virtual mass sensation," in Proc. ACM SIGGRAPH Emerging Technol., 2007, Art. no. 8

[14] K. Minamizawa, D. Prattichizzo, and S. Tachi, "Simplified design of haptic display by extending one-point kinesthetic feedback to multipoint tactile feedback," in Proc. IEEE Haptics Symp., 2010, pp. 257-260.

[15] C. Pacchierotti, G. Salvietti, I. Hussain, L. Meli, and D. Prattichizzo, "The hRing: A wearable haptic device to avoid occlusions in hand tracking," in Proc. IEEE Haptics Symp., Apr. 2016, pp. 134-139.

[16] M. Gabardi, M. Solazzi, D. Leonardis, and A. Frisoli, "A new wearable fingertip haptic interface for the rendering of virtual shapes and surface features," in Proc. IEEE Haptics Symp., Apr. 2016, pp. 140-146.

[17] A. Moscatelli, et al., "The change in fingertip contact area as a novel proprioceptive Cue," Current Biol., vol. 26, no. 9, pp. 11591163,2016

[18] S. J. Lederman and R. L. Klatzky, "Relative availability of surface and object properties during early haptic processing," J. Exp. Psychology: Human Perception Performance, vol. 23, no. 6, 1997, Art. no. 1680.

[19] W. M. B. Tiest and A. M. Kappers, "Cues for haptic perception of compliance," IEEE Trans. Haptics, vol. 2, no. 4, pp. 189-199, Oct.-Dec. 2009.

[20] M. A. Srinivasan and R. H. LaMotte, "Tactile discrimination of softness," J. Neurophysiology, vol. 73, no. 1, pp. 88-101, Jun. 1995.

[21] A. Bicchi, E. P. Scilingo, and D. De Rossi, "Haptic discrimination of softness in teleoperation: The role of the contact area spread rate," IEEE Trans. Robot. Autom., vol. 16, no. 5, pp. 496-504, Oct. 2000.

[22] M. Bianchi, E. Battaglia, M. Poggiani, S. Ciotti, and A. Bicchi, "A wearable fabric-based display for haptic multi-cue delivery," in Proc. IEEE Haptics Symp., 2016, pp. 277-283.

[23] M. Bianchi, E. P. Scilingo, A. Serio, and A. Bicchi, "A new softness display based on bi-elastic fabric," in Proc. World Haptics Conf., 2009, pp. 382-383.

[24] M. Bianchi, A. Serio, E. P. Scilingo, and A. Bicchi, "A new fabricbased softness display," in Proc. IEEE Haptics Symp., 2010, pp. $105-112$.

[25] A. Serio, M. Bianchi, and A. Bicchi, "A device for mimicking the contact force/contact area relationship of different materials with applications to softness rendering," in Proc. IEEE/RSJ Int. Conf. Intell. Robots Syst., Nov. 2013, pp. 4484-4490.

[26] M. Bianchi and A. Serio, "Design and characterization of a fabricbased softness display," IEEE Trans. Haptics, vol. 8, no. 2, pp. 152163, Apr. 2015.

[27] F. Kimura, A. Yamamoto, and T. Higuchi, "Development of a 2-DoF softness feeling display for tactile tele-presentation of deformable surfaces," in Proc. IEEE Int. Conf. Robot. Autom., May 2010, pp. 1822-1827. 
[28] M. Bianchi, "A fabric-based approach for wearable haptics," Electronics, vol. 5, no. 3, 2016, Art. no. 44. [Online]. Available: http:// www.mdpi.com/2079-9292/5/3/44

[29] S. Condino, et al., Tactile Augmented Reality for Arteries Palpation in Open Surgery Training. Cham, Switzerland: Springer, 2016, pp. 186-197. [Online]. Available: http://dx.doi.org/10.1007/9783-319-43775-0_17

[30] J. D. Brown, M. Ibrahim, E. D. Chase, C. Pacchierotti, and K. J. Kuchenbecker, "Data-driven comparison of four cutaneous displays for pinching palpation in robotic surgery," in Proc. IEEE Haptics Symp., 2016, pp. 147-154.

[31] M. L. D'Angelo, et al., "An integrated approach to characterize the behavior of a human fingertip in contact with a silica window," IEEE Trans. Haptics, vol. 10, no. 1, pp. 123-129, Jan.-Mar. 2017.

[32] S. J. Lederman and R. L. Klatzky, "Hand movements: A window into haptic object recognition," Cognitive Psychology, vol. 19, no. 3, pp. 342-368, 1987.

[33] G. A. Gescheider, "Psychophysics: Method," Theory and Application. Hillsdale, NJ, USA: Erlbaum, 1985.

[34] A. Moscatelli, M. Mezzetti, and F. Lacquaniti, "Modeling psychophysical data at the population-level: The generalized linear mixed model," J. Vis., vol. 12, no. 11, 2012, Art. no. 26.

[35] A. Moscatelli, A. Naceri, and M. O. Ernst, "Path integration in tactile perception of shapes," Behavioural Brain Res., vol. 274, pp. 355364, Nov. 2014

[36] S. Ullrich and T. Kuhlen, "Haptic palpation for medical simulation in virtual environments," IEEE Trans. Vis. Comput. Graph., vol. 18, no. 4, pp. 617-625, Apr. 2012.

[37] T. R. Coles, N. W. John, D. Gould, and D. G. Caldwell, "Integrating haptics with augmented reality in a femoral palpation and needle insertion training simulation," IEEE Trans. Haptics, vol. 4, no. 3, pp. 199-209, Jul.-Sep. 2011.

[38] C. Pacchierotti, S. Sinclair, M. Solazzi, A. Frisoli, V. Hayward, and D. Prattichizzo, "Wearable haptic systems for the fingertip and the hand: Taxonomy, review, and perspectives," IEEE Trans. Haptics, vol. 10, no. 4, pp. 580-600, Oct.-Dec. 2017.

[39] W. Tiest and A. M. L. Kappers, "Cues for haptic perception of compliance," IEEE Trans. Haptics, vol. 2, no. 4, pp. 189-199, Oct.-Dec. 2009

[40] E. P. Scilingo, M. Bianchi, G. Grioli, and A. Bicchi, "Rendering softness: Integration of kinaesthetic and cutaneous information in a haptic device," IEEE Trans. Haptics, vol. 3, no. 2, pp. 109-118, Apr.-Jun. 2010.

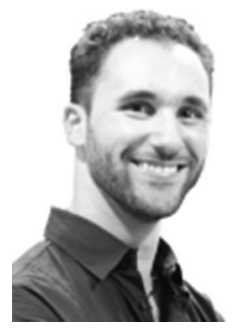

Simone Fani received the bachelor's degree in information engineering, curriculum in automatic systems and industrial automation from the University of Siena, in 2011 and the master's degree in robotics and automation engineering from the University of Pisa, in 2015. He is currently working toward the PhD degree at the Research Center "E. Piaggio", University of Pisa. His research interests include design and validation of haptic interfaces for prosthetics and tele-operation systems, medical and rehabilitation robotics, and mathematical modeling of the sense of touch. He is a student member of the IEEE.

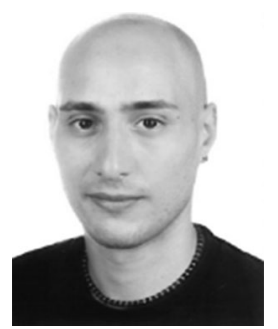

Simone Ciotti received the bachelor's degree in information engineering and the master's degree in robotics and automation engineering from the University of Pisa, in 2010 and 2015, respectively. $\mathrm{He}$ is currently working toward the $\mathrm{PhD}$ degree at the Research Center "E. Piaggio", University of Pisa. His research interests include mathematical modeling of the sense of touch, design and validation of haptic interfaces for augmented and virtual reality, medical, and rehabilitation robotics. $\mathrm{He}$ is a student member of the IEEE.

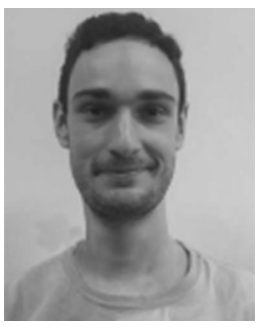

Edoardo Battaglia received the bachelor's degree in mechanical engineering and the master's degree in robotics and automation engineering from the University of Pisa, in 2009 and 2013, respectively. $\mathrm{He}$ is currently working toward the PhD degree at the Research Center "E. Piaggio", University of Pisa. His research interests include design and validation of haptic interfaces (with a focus on wearable applications), medical and rehabilitation robotics, and mathematical modeling of the sense of touch and human manipulation. He is a student member of the IEEE.

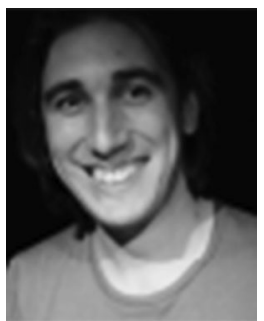

Alessandro Moscatelli received the medical doctor degree and the postgraduate master's degree from the University of Rome "Tor Vergata", in 2006 and 2008, respectively, and the PhD degree in neuroscience from the University of Rome "Tor Vergata", in 2010. In 2007, he was a research fellow in the Santa Lucia Foundation. Currently, he is working as a post doc in the Department of System Medicine, University of Rome "Tor Vergata", and as a researcher in the Department of Neuromotor Physiology of the Santa Lucia Foundation. He is a co-investigator in the European projects "The Hand Embodied" and "WearHap". From 2011 to 2015, he worked as post doc in the Department of Cognitive Neuroscience and CITEC, Bielefeld University. His current research topic is the behavioral and neural correlates of haptic perception. His research interests include the representation of space from tactile motion cues, and results of these studies shows the capacity of the tactile system to integrate the velocity-signal over time. As a further research topic, he is currently studying the integration of proprioception and touch in the perception of object motion. He is familiar with psychophysical methods; he developed an R package for the estimate of psychophysical parameters in mixed model framework.

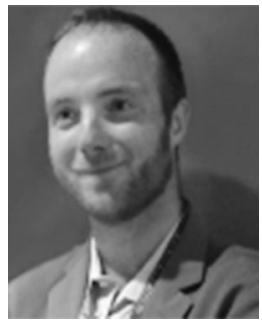

Matteo Bianchi received the BS and MS (cum laude) degrees in biomedical engineering from the University of Pisa, Italy, in 2004 and 2007, respectively, and the PhD degree in automatics, robotics, and bioengineering from the University of Pisa, in 2012. Currently, he is working as an assistant professor at the University of Pisa - Department of Information Engineering - Centro di Ricerca "E. Piaggio". From January to June 2011, he worked as a visiting student at the Laboratory for Computational Sensing and Robotics, Johns Hopkins University (Baltimore, Maryland). His research interests include haptic interface design, with applications in medical robotics and assistive/ affective human-robot interaction; human and robotic hands: optimal sensing and control; human-inspired control for soft robots; and psychophysics and mathematical modelling of the sense of touch and human manipulation. $\mathrm{He}$ is an author of contributions to international conferences and journals. He serves as a reviewer and member of the editorial board and organizing committee of international journals and conferences. He is coeditor of the book Human and Robot Hands, Springer International Publishing. In 2010, he was a finalist for the Best Student Paper and Best Paper Award at the IEEE Haptics Symposium; in 2012, he was awarded with the JCTF Novel Technology Paper Award at the IEEE/RSJ IROS Conference and in 2014 with the Best Paper Award (poster category) at the Eurohaptics Conference. In 2015, he was awarded with the Best Oral Presentation Award at Automatica.it 2015 Conference organized by the Italian Society of Researchers in Automation and with the Meritorious Service Award for his work as a Reviewer for the IEEE Transactions on Haptics. In 2016, he was awarded with the Best Paper Award at the IEEE Haptics Sympoisum. In 2017, he was finalist for the Best Paper Award with two manuscripts, one of them was also finalist for the Best Student Paper Award at the IEEE Worldhaptics conference. He is a member of the IEEE and serves as cochair of the IEEE Robotics and Automation Society Technical Committee on Robotic Hand Grasping and Manipulation. 